\title{
On an Alternate Construction Method for Generating Spidrons and New Tiling Patterns Generated by them
}

\author{
T. Gangopadhyay \\ $\mathrm{XLRI}$ \\ C.H.Area(E), Jamshedpur, \\ India
}

\begin{abstract}
Spidrons have been constructed by repeatedly connecting the alternate vertices of a regular polygon. In the present paper an alternate construction is presented. Also new polygonal designs and tiling patterns are created using these spidrons
\end{abstract}

\section{General Terms}

Tiling, Algorithm, Turbo C++, Program

\section{Keywords}

Spidron, polygon, isosceles

\section{INTRODUCTION}

In earlier papers, the present author has studied tiling with stars having an odd number of vertices (Gangopadhyay([3],[4]). In the present paper, the author studies tiling patterns using a fractalesque structure called spidron, first studied by Erdely[2]. An n-part spidron is a figure in plane geometry consisting entirely of an alternating sequence of two isosceles triangles, each with its own base angles[2], [6], [8]. The two triangles are juxtaposed in such a way that together they from a third bigger triangle. The sequence is then drawn at an appropriate angle on a smaller scale and the process is repeated ad infinitum. A regular nsided polygon can be split into n n-part spidrons[7]. A 6-part spidron is displayed in Figure 1. Erdely[2], has called the same structure a semispidron. The usual method of constructing spidrons consists of starting with a regular polygon, connecting its alternate vertices and producing a smaller version of the regular polygon inside. The process is then repeated for the smaller polygon and so on ad infinitum. This divides the polygon into spidrons.

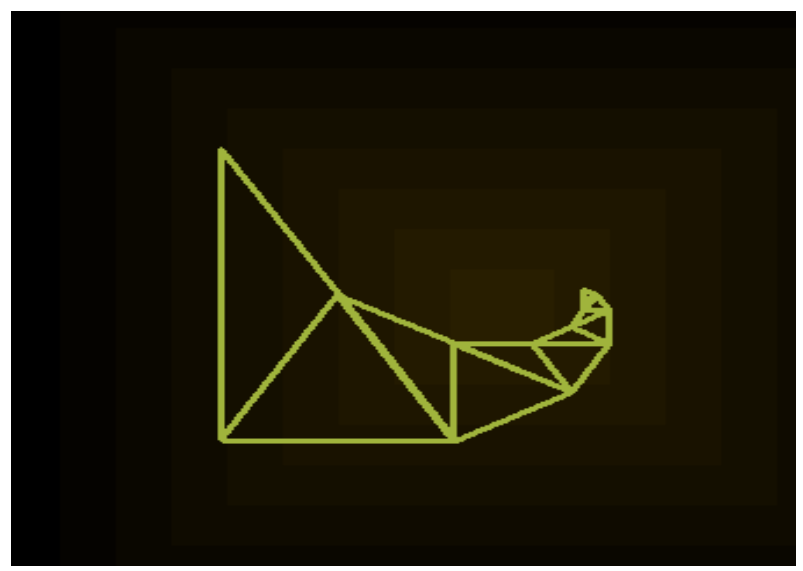

Fig 1 : A six-part spidron

In the present paper, the author presents an alternate way of constructing individual n-part spidrons and then algorithmically juxtapose them to produce a regular n-sided polygon. In a way the new method uses a bottoms-up approach as compared to the usual method which uses a topdown one. This preserves the individuality of the spidrons from the outset and allows dazzling designs to be made inside the regular polygon by using different colors for different polygons.

n-part spidrons have been largely studied for $n=6$ and $8[5]$ The present method illustrates n-part spidrons for other values of $\mathrm{n}$ as well.

Apart from generating nested spidrons inside a regular polygon, the paper also explores diverse tiling techniques using these. These are primarily the distinctive features of this paper.

\section{THE ALGORITHM}

The algorithm is best explained in terms of Figure 2, which depicts two juxtaposed isosceles triangles, this being the basic building block of a spidron. Let the base angle $\mathrm{ABC}$ have value ag and let $A B$ have length $s$. Then $A C=C D=A B=s$ and angle $\mathrm{ACB}=\mathrm{ag}$. Also angle $\mathrm{CAD}=$ angle $\mathrm{ADC}$.From this it follows that angle $\mathrm{CAD}=$ angle $\mathrm{ACD}=\mathrm{ag} / 2$. Also $\mathrm{Bc}=2 \mathrm{~s}(\cos (\mathrm{ag}))$ and $\mathrm{AD}=2 \mathrm{~s}(\cos (\mathrm{ag} / 2))$. With this it is easy to construct the two triangles in Figure 2. It is to be noted that for $\mathrm{n} n$-part spidrons to be nested inside an $\mathrm{n}$-sided regular polygon, ag=360/n.

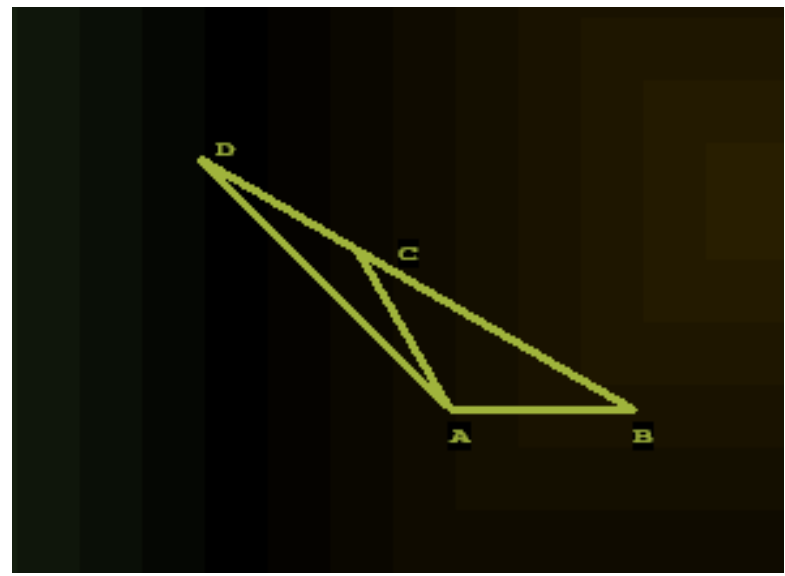

Fig 2 : The first iteration

For the recursive step, one refers to Figure 3, in which the next iteration is shown. It can be easily calculated that $\mathrm{BE}=$ $\mathrm{s}(\cos (\mathrm{ag}) / \cos (\mathrm{ag} / 2))$. Accordingly $\mathrm{s}$ is replaced by this value before the recursive call. 


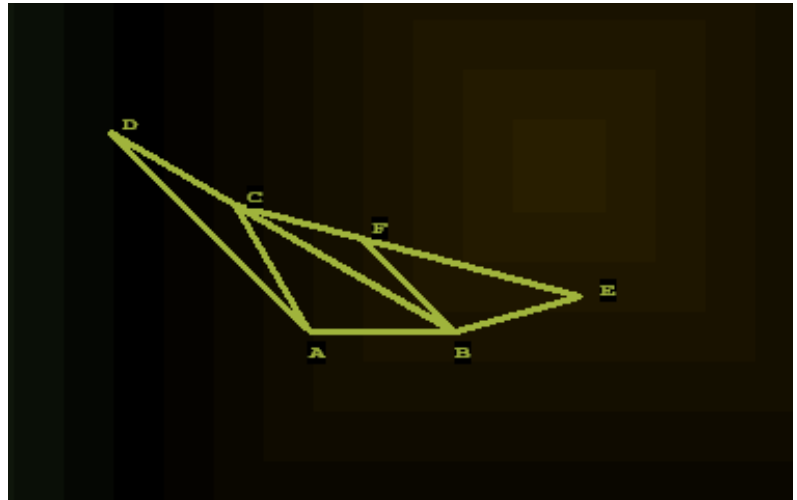

Fig 3 : The second iteration

Figure 4 depicts a 12-part spidron after 8 iterations.

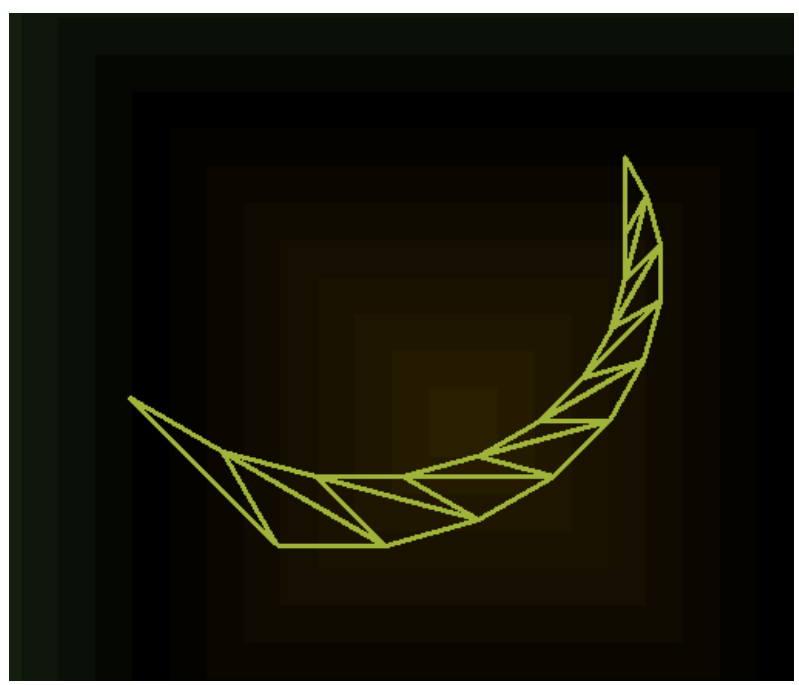

Fig 4 : A 12-part spidron after 8 iterations

In the next section one submits a programming code in Visual $\mathrm{C}++$ that captures the algorithm and generates its output.

\section{THE CODE}

The code uses a function spid which is declared first. The function has two parameters $-\mathrm{s}$, which gives the value of the two equal sides of the first isosceles triangle and 1, which specifies the number of recursive iterations. The function draws a single n-part spidron. There is also another function, polyspidron, which draws $\mathrm{n} n$-part spidrons in such a way that they are nested inside an n-sided regular polygon. This is achieved by calculating the appropriate angle between two consecutive spidrons. The spidrons are then colored using different colors thereby creating a spiral like structure. The functions $\mathrm{fd}, \mathrm{rt}, \mathrm{lt}, \mathrm{pu}$ and $\mathrm{pd}$ are adapted from turtle graphics[1] and have their usual connotations. Fd draws a line od specified length, rt and lt respectively rotates the pen right and left by a specified angle, pu puts the pen up(no drawing) and pd puts the pen down. The various functions and the code are given below:

int $\mathrm{n}=12$;

float ang $=90, p x, p y, p s=1$;

void fd(float dist)

\{float $\mathrm{hx}=\cos (\mathrm{ang} * 3.1415926536 / 180)$;

float hy $=\sin ($ ang $* 3.1415926536 / 180)$; float $\mathrm{nx}=\mathrm{px}-\mathrm{hx} *$ dist;

float ny=py-hy*dist;

if(ps!=1)goto label;

line(px,py,nx,ny);

label:px=nx;py=ny;

\}

float rt(float 1$)$

$\{$ ang+=l;

return ang;\}

float lt(float 1$)$

\{ ang-=l;

return ang; $\}$

void pu()

$\{\mathrm{ps}=0 ;\}$

void $\mathrm{pd}()$

$\{\mathrm{ps}=1 ;\}$

void spid(float s,int l)

\{float a,b,c;a=px,b=py,c=ang;float ag=360/n;

if $(1==0)\{\operatorname{rt}(90) ; f d(s) ; \operatorname{lt}(180-a g) ; f d(s)$;

$\mathrm{fd}(2 * \mathrm{~s} * \cos (\mathrm{ag} * 3.14 / 180))$;

lt $(180-\mathrm{ag} / 2) ; \mathrm{fd}(2 * \mathrm{~s} * \cos (\mathrm{ag} / 2 * 3.14 / 180))$;

lt(180-ag/2);fd(s);rt(180-ag);

$\mathrm{pu}() ; \mathrm{fd}(\mathrm{s} * 2 * \cos (\mathrm{ag} * 3.14 / 180)) ; \mathrm{pd}()$;

return;\}

spid (s,0); $\operatorname{lt}(90+a g+a g / 2)$;

spid $(\mathrm{s} * \cos (\mathrm{ag} * 3.14 / 180) / \cos (\mathrm{ag} / 2 * 3.14 / 180), 1-1)$;

$\mathrm{px}=\mathrm{a}, \mathrm{py}=\mathrm{b}, \mathrm{ang}=\mathrm{c}$;

\}

void polyspidron(float s,int l)

$\{$ for $($ int $\mathrm{i}=0 ; \mathrm{i}<\mathrm{n} ; \mathrm{i}++)$

\{ setcolor(i); spid (s,1);rt(90+180/n);

pu();fd(2*s*cos(180/n*3.14/180));pd();lt(90+360/n+180/n);

\}

void main()

\{

initwindow $(1000,800)$;

setlinestyle(SOLID_LINE,0,THICK_WIDTH);

float $s=70 ; p x=380, p y=620$;

polyspidron(s,16);

getch();

closegraph();

\} 
The output of the sample code is illustrated in Figure 3, a regular dodecahedron showning the spiralling nested spidrons.

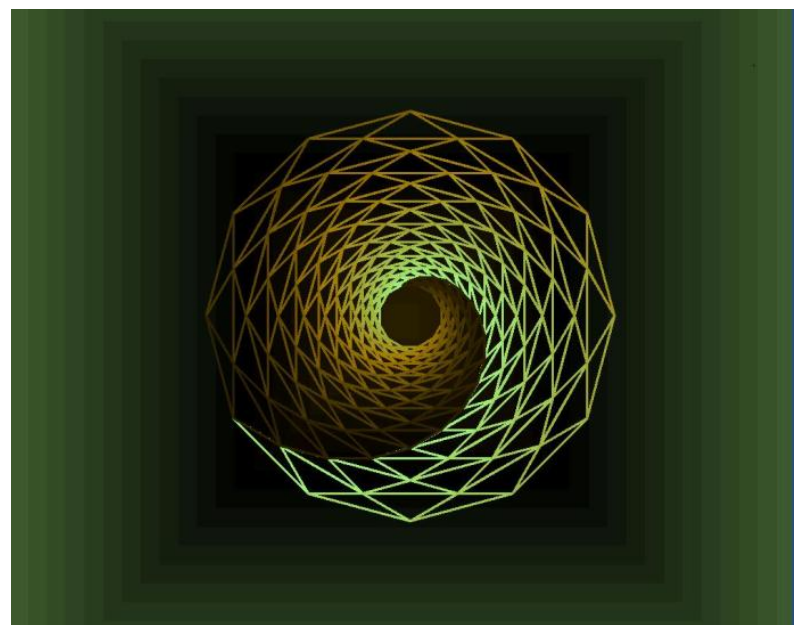

Fig 5 : Output of the sample code

\section{INSCRIBING A REGULAR POLYGON USING 6-PART SPIDRONS}

Using n Six-part spidrons one can also construct other symmetric designs that inscribe a regular polygon with $n$ sides. For this, the variable ag in the function spid has to be set to $360 / 6$ or 60 . Also the function polyspidron has to be changed to a function polyspidron 1 which is defined below:

void polyspidron 1 (float s,int 1 )

$\{$ for(int $\mathrm{i}=0 ; \mathrm{i}<\mathrm{n} ; \mathrm{i}++)$

\{ spid(s,5); rt(90); fd(s);

$\operatorname{rt}(90-(2 * n-4) * 90 / n) ;\}$

\}

Figures 6 and 7 depict the respective outputs for $n=6$ and $n=9$.

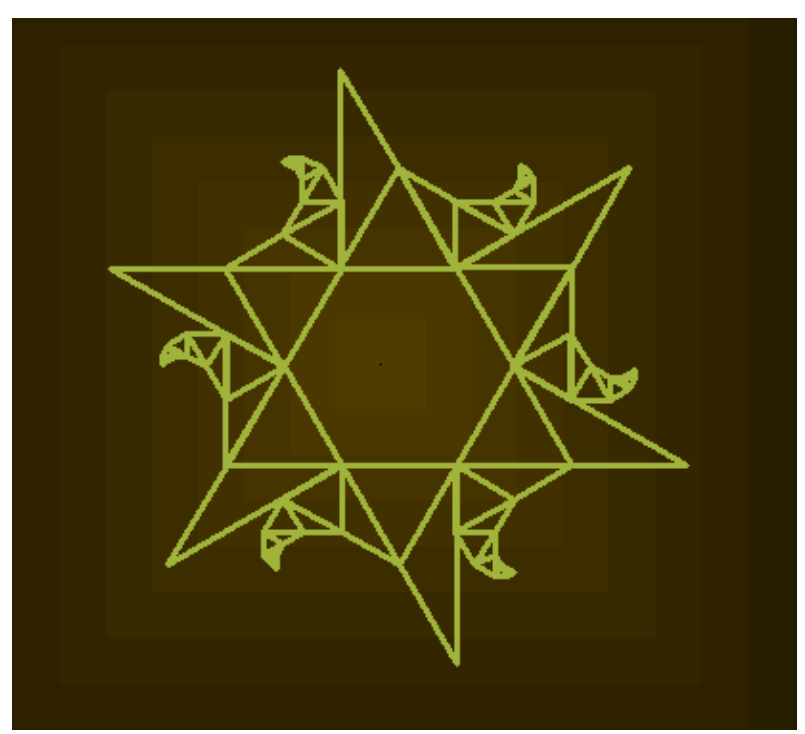

Fig. 6 : Output of the modified code for $n=6$

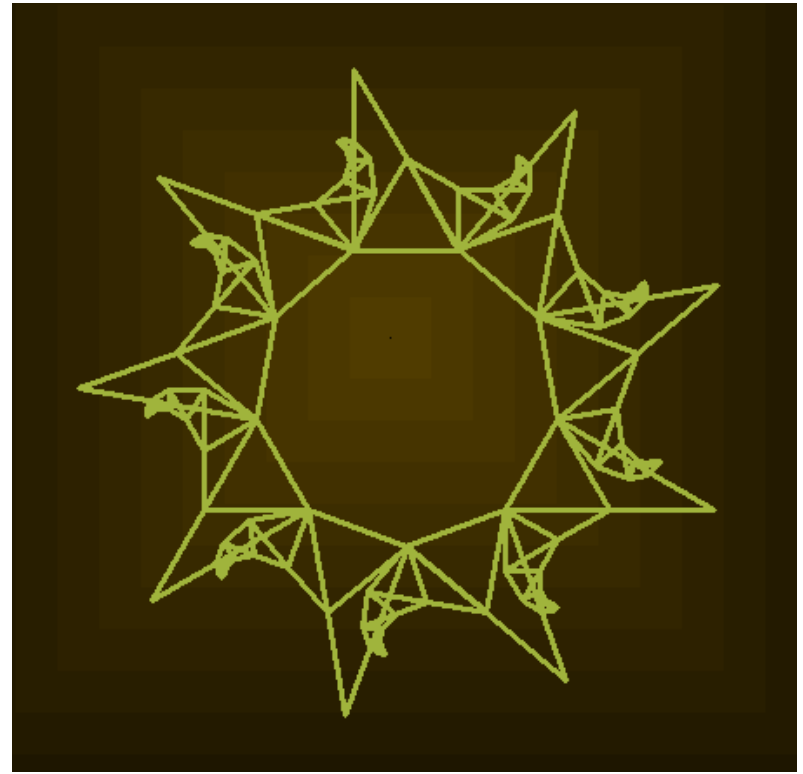

Fig. 7 : Output of the modified code for $n=9$

It can be easily observed from figures 6 and 7 that the new design will always inscribe an $\mathrm{n}$-sided regular polygon.

\section{TILING THE PLANE USING SPIDRONS}

Both the nested spidrons of section 3 and the new inscribed polygonal designs of section 4 can be used for tiling the plane. Figures 8 and 9 depict such tiling for $n=6$ and 8 respectively. To produce the output in Figure 9, the calling of the function polyspidron in the portion main() should be replaced by the following segment of code.

for(int $\mathrm{i}=0 ; \mathrm{i}<8 ; \mathrm{i}++)$

for(int $j=0 ; j<7 ; j++)$

$\{\mathrm{px}=152 * \mathrm{i}, \mathrm{py}=152 * \mathrm{j}$;

for(int $\mathrm{i}=0 ; \mathrm{i}<\mathrm{n} ; \mathrm{i}++)$ polyspidron $(20,16)$; $\}$

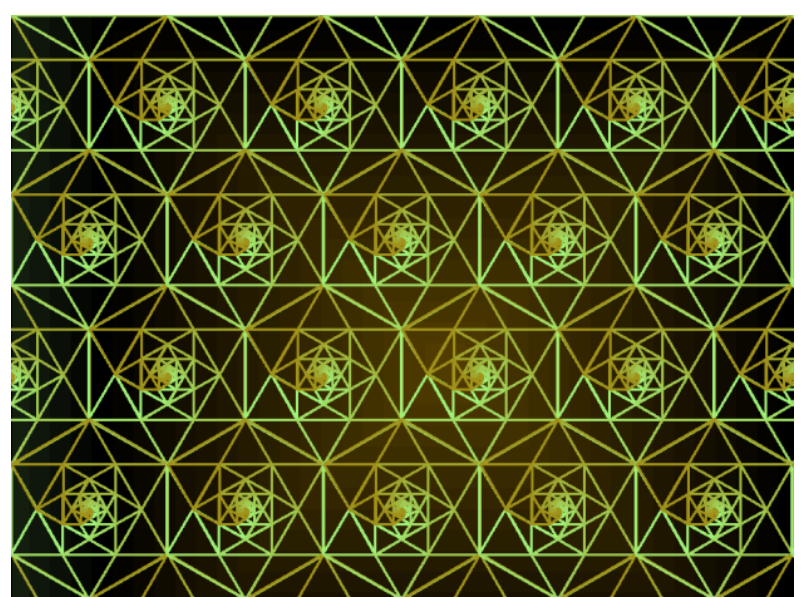

Fig. 8 : Tiling with nested spidrons for $n=6$

5. 


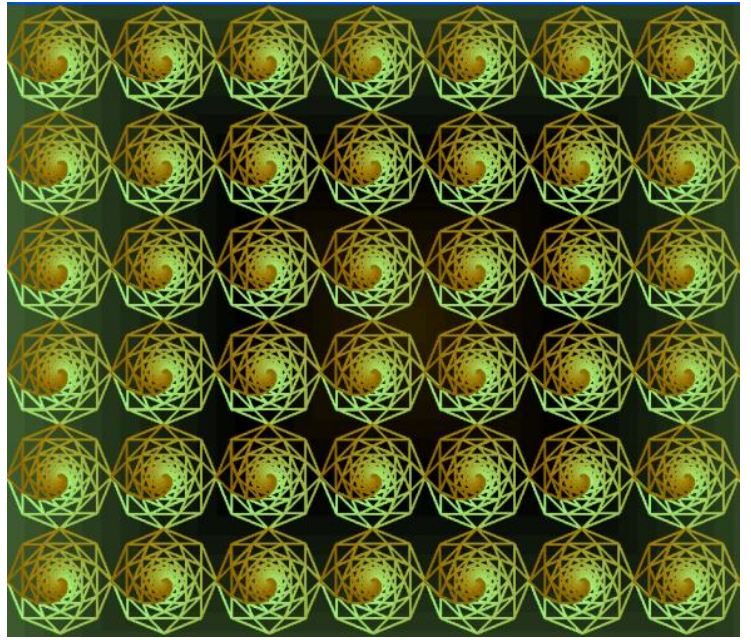

Fig. 9 : Tiling with nested spidrons for $\mathbf{n}=8$

Figures 10 to 13 explore various tiling patterns created with the new inscribed polygonal designs for $n=3,4,5$ and 8 . For instance, to produce the output in Figure 13, the calling of the function polyspidron in the portion main() should be replaced by the following segment of code

for(int $\mathrm{i}=0 ; \mathrm{i}<8 ; \mathrm{i}++)$

for(int $j=0 ; j<7 ; j++)$

$\{\mathrm{px}=126 * \mathrm{i}, \mathrm{py}=126 * \mathrm{j}$;

for(int $\mathrm{i}=0 ; \mathrm{i}<\mathrm{n} ; \mathrm{i}++)$ polyspidron $1(20,16) ;\}$

Here polyspidron 1 is the function that has been defined in section 4.

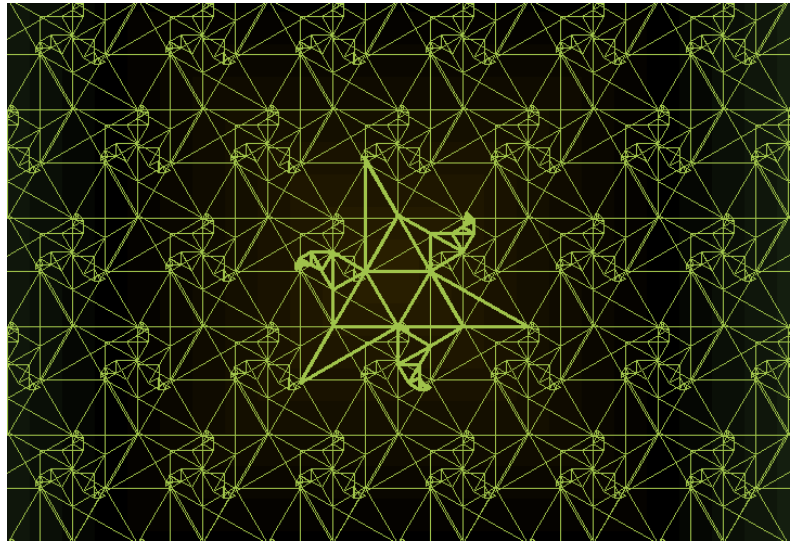

Fig.10. Tiling pattern using the new design for $n=3$

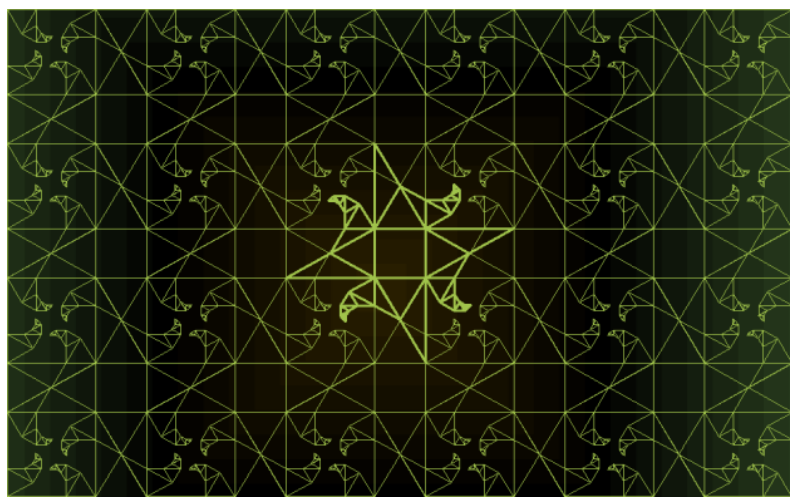

Fig. 11 : Tiling pattern using the new design for $n=4$

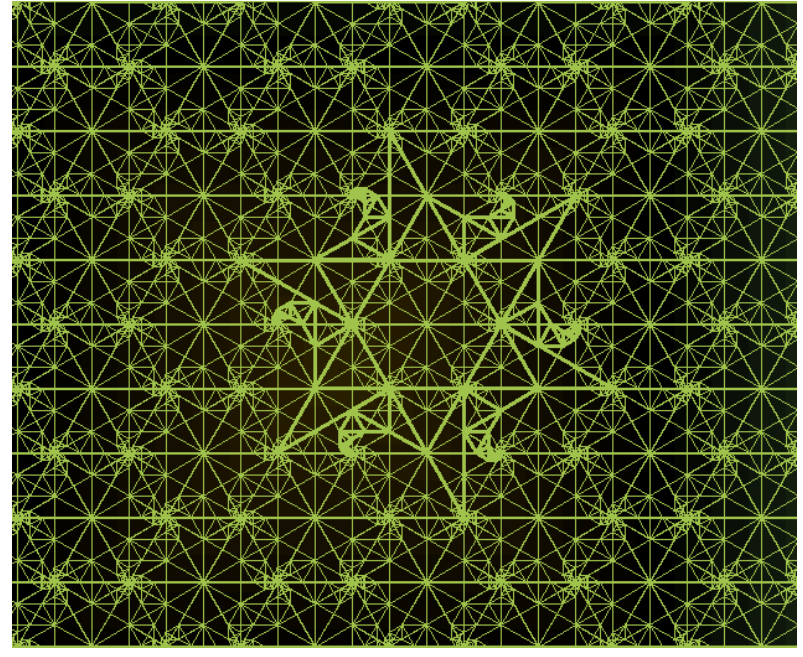

Fig. 12 : Tiling pattern using the new design for $n=6$

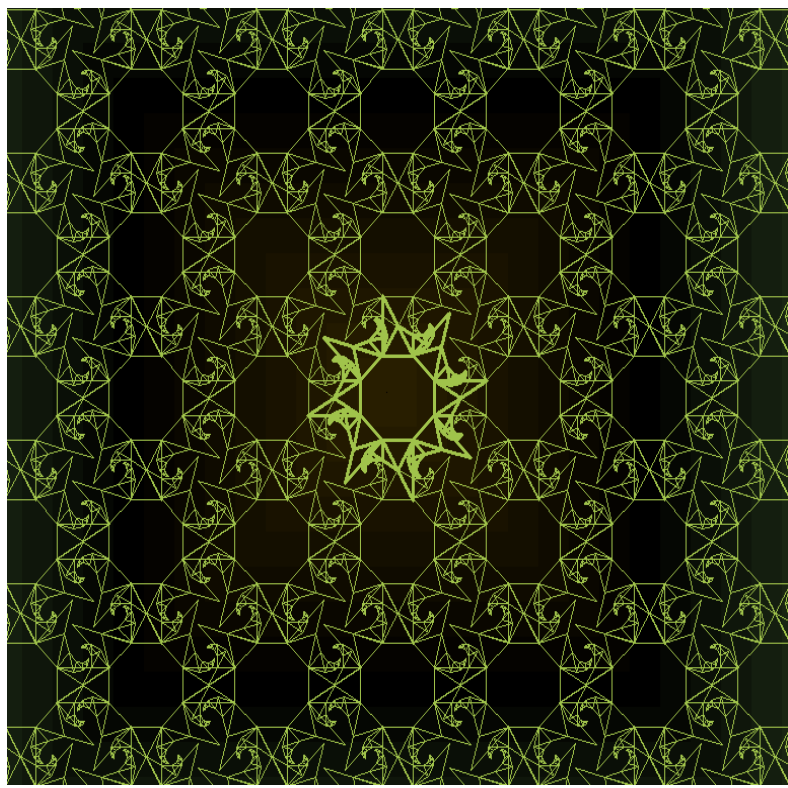

Fig. 13 : Tiling pattern using the new design for $n=8$

\section{CONCLUSION}

This paper presents an alternate method for constructing spidrons which are used to construct diverse polygonal designs and also explores various tiling designs using the same. In subsequent studies one will further study the effect of superimposing two or more such polygonal designs on tiling patterns. One will also study aperiodic tiling patterns created with polygonal designs using an odd number of spidrons. These are the aspects that would be explored in future work.

\section{ACKNOWLEDGMENTS}

The author wishes to acknowledge his debt to the referee(s) for their constructive suggestions and encouragement

\section{REFERENCES}

[1] Abelson and diSessa, Turtle Geometry, MIT Press, 1992

[2] Erdely,D.http://www.bridgesmathart.org/art exhibits/bridges2007/erdely.html.

[3] Gangopadhyay, T. On Tiling Patterns Involving Islamic Rosettes with an Odd Number of Vertices, International journal of Computer Applications, 69(2013) 9-14.. 
[4] Gangopadhyay, T. Further Tiling Patterns Involving Islamic Rosettes with an Odd Number of Vertices, International journal of Computer Applications, 71(2013)36-41.

[5] Jacques, F. http://polyspidrons.over-blog.com/article4823990.html.
[6] Peterson, I. "Swirling Seas, Crystal Balls". ScienceNews.org. Archived from the original on February 28, 2007. Retrieved 2007-02-14.

[7] Stenzhorn, S. Mathematical description of Spidrons ,http://stefanstenzhorn.com/Spidrons.

[8] https://en.wikipedia.org/wiki/Spidron. 